\title{
TEACHING STRATEGIES IN THE CLASSROOM
}

\section{ESTRATEgIAS PEDAGógICAS EN EL AULA DE CLASE}

\author{
PhD. Torcoroma Velásquez Pérez, MSc. Edwin Edgardo Espinel Blanco \\ MSc(c). Gustavo Guerrero Gómez
}

Universidad Francisco de Paula Santander Ocaña, Facultad de Ingenierías Vía Acolsure Sede Algodonal, Ocaña, Norte de Santander, Colombia. Teléfono: (+57) 569 0088- Ext. 182.

E-mail: tvelasquezp@ufpso.edu.co, eespinelb@ufpso.edu.co, gguerrerog@ufpso.edu.co

\begin{abstract}
Resumen: Este trabajo tiene como propósito la evaluación de la adopción del modelo Constructivista Social, con el fin de validar la hipótesis de que con el avance de los semestres académicos aumenta la incorporación de estrategias pedagógicas que permiten la mejor adopción del enfoque Constructivista Social por parte de los docentes de la UFPSO. El estudio se plantea mediante una metodología descriptiva-evaluativa teniendo en cuenta la población de 362 docentes entre planta y catedráticos, de la cual se toma una muestra de 76 profesores. Se definen como variables el Rendimiento Académico y las Estrategias Pedagógicas, se generan varios inter grupos con cada facultad, se contempla un plan que incluye el análisis comparativo y correlación de Pearson entre facultades.
\end{abstract}

Palabras clave: Estrategias Pedagógicas, Modelo Pedagógico, Rendimiento Académico

Abstract: This paper aims to evaluate the adoption of the Social Constructivist model, in order to validate the hypothesis that with the advancement of the academic semesters increases the incorporation of pedagogical strategies that allow the best Social Constructivist approach adopted by the teachers of UFPSO. This study presents a descriptive-evaluative methodology taking into account the population of 362 teachers and professors from plant from which a sample of 76 teachers is taken. Variables are defined as academic performance and teaching strategies, inter several groups are generated by each faculty, a plan that includes comparative analysis and Pearson correlation between powers is contemplated.

Keywords: Instructional Strategies, Teaching Model, Academic Performance

\section{INTRODUCCIÓN}

La Universidad Francisco de Paula Santander Ocaña, plantea como enfoque pedagógico el Constructivismo Social (PEI, 2.012), el objetivo de la investigación presentada es evaluar la adopción de este modelo por parte de los docentes de la Universidad Francisco de Paula Santander Ocaña UFPSO. Inicialmente se parte del concepto de modelo, entendido como una representación mental del mundo real que orienta toda acción (Duran,
Velásquez, \& Cárdenas, 2013), y se convierte en una herramienta conceptual que permite orientar y entender un evento, una realidad, una acción, entre otros.

En la pedagogía se estudian los procesos de formación, abriendo espacios para la reflexión del proceso de enseñanza-aprendizaje y producción del conocimiento en todas las áreas disciplinares y transdisciplinares (Duran, Velásquez, \& Cárdenas, 2013); por tanto, la pedagogía implica entender los 
diferentes modelos pedagógicos y su relación con las prácticas educativas y docentes.

Como se cita en (Velásquez, Pérez et al, 2015) un modelo pedagógico "es la representación de las relaciones que predominan en el acto de enseñar, es también un paradigma que puede coexistir con otros y que sirve para organizar la búsqueda de nuevos conocimientos en el campo de la pedagogía" (Flórez, 1994); de la misma manera y de acuerdo a lo planteado por el Ministerio de Educación Nacional de Colombia MEN, "el modelo pedagógico es la relación flexible, dinámica, dialéctica, entre contenidos, fines, maestros, alumnos y métodos. El modelo pedagógico es un constructo teórico y de interacción en un contexto específico que alimenta una perspectiva futura de formación y que se construye para concretar propósitos e intencionalidades referidas a un proyecto de sociedad, de cultura y de educación”.

El constructivismo es una teoría que "propone que el ambiente de aprendizaje debe sostener múltiples perspectivas o interpretaciones de realidad, construcción de conocimiento, actividades basadas en experiencias ricas en contexto" (JONASSEN, 1994). La base de esta teoría es generar conocimiento en actividades propias, con fin de que sea posible apropiarse del tema con tareas familiares.

El modelo denominado "entornos de aprendizaje constructivista" se identifica por tener características del aprendizaje constructivista (JONASSEN, 1994), estas características están enfocadas a la relación que debe existir entre el diario vivir y el proceso de enseñanza. En estos entornos se incorporan múltiples representaciones de la realidad y entornos sociales para la apropiación del conocimiento, representando la complejidad del mundo real permitiendo la retroalimentación del conocimiento dentro de la reproducción del mismo, o visto como una fuente de descubrimiento del conocimiento humano que implica tomar los saberes anteriores para generar procesos de abstracción- concreción sistemáticos contribuyendo esto con los cambios de actitud en las personas en relación con la construcción intelectual y sus aplicaciones (Umanzor , 2.011).

Con el propósito de realizar procesos de autoevaluación en la universidad Francisco de Paula Santander Ocaña, se han realizado al interior de programas como la Ingeniería de Sistemas varios estudios a través de técnicas inteligentes como las redes neuronales artificiales (Araque et al., 2013) y los sistemas basados en conocimiento que buscaban realizar autoevaluación del programa (Velásquez, 2010) y apoyar procesos de deserción como sistemas inteligentes de Orientación vocacional (Velásquez, et al, 2014), de igual manera hacer reflexiones sobre los aspectos ontológicos y epistemológicos del programa (Velásquez, et al, 2015).

Continuando los procesos investigativos que le aporten al mejoramiento de los programas académicos y teniendo presente los conceptos asociados con el modelo pedagógico, se proponen como fases de la investigación, primero conocer el modelo pedagógico planteado por la universidad desde su Proyecto Educativo Institucional-PEI, permitiendo conocer las estrategias para la adopción del modelo, una siguiente fase consistió en el diseño de los instrumentos necesarios para realizar la investigación y después de aplicar los instrumentos se continúa con el análisis de la información a través de la triangulación entre los grupos seleccionados que permitieron realizar la evaluación planteada, teniendo presente que estos resultados son preliminares ya que se realizaron con evaluaciones parciales y no finales del semestre lo que propone realizar un comparativo al final de semestre para conocer si la tendencia continua.

\section{MODELO TEÓRICO-EPISTEMOLÓGICO}

El modelo constructivista social tiene múltiples enfoques, no solamente debemos observar el rol que toma el docente y como este se desempeña en clase sino que también debemos observar cómo el estudiante asimila estos conocimientos, por ello se debe adoptar una metodología que nos permita analizar diferentes puntos de vista como por ejemplo, evaluar la importancia y condición del nuestro modelo educativo mediante un cuidadoso estudio y minucioso enfoque.

Se inicia observando cómo el docente implementa el modelo constructivista en sus clases, se analiza la percepción y asimilación de conceptos que tiene el estudiante con la metodología que el docente implementa y a continuación se describen las conclusiones productos de la observación y del análisis. Este enfoque permite tener una concepción de cómo es adoptado el modelo constructivista social en las aulas de clases de diferentes facultades; este estudio permitirá evaluar y crear estrategias que permitan mejorar la 
implementación de este modelo adoptado por nuestra alma mater.

Para realizar este proyecto de investigación se acude al tipo de investigación cuantitativa, utilizando métodos tradicionales para el análisis de dicha información. Teniendo en cuenta que los elementos de la investigación tienen una relación cuya naturaleza puede ser representada por un modelo numérico. Con los resultados obtenidos se podrá probar o refutar la hipótesis sin ambigüedad; de igual forma se propone la investigación descriptiva que involucra las características y situaciones de interés de la población, para poder ordenar los resultados de las observaciones, variables de los hechos, rasgos y factores. Es decir un instrumento estadístico para medir el impacto y las estrategias del modelo pedagógico aplicado en la UFPSO.

Se tomó de la población total (OARC, 2015) de docentes 362 (DOC_UFPSO) y estudiantes 6339 (EST_UFPSO) de la Universidad Francisco de Paula Santander Ocaña cuatro grupos, así: Facultad de Ciencias Agrarias, otro grupo Facultad de Ciencias Económicas y Administrativas, La Facultad de Ingeniería y el otro grupo de la Facultad de Educación, Artes y Humanidades. Se utilizó la muestra estadística para los docentes con un nivel de confianza del $95 \%$, la proporción de aceptación y de rechazo del $50 \%$ y un error menor al 0.1, donde se calcula una muestra de 76 docentes.

Teniendo en cuenta la cantidad de estudiantes por facultad se determina la muestra de 16 docentes por Ciencias Agrarias y del Ambiente, 19 de Ciencias Administrativas y Económicas, 20 de Ingenierías y 21 de Educación, Artes y Humanidades. Por cada docente se aplican tres instrumentos, una encuesta para el docente, una para los estudiantes de ese docente que puede ser 15 estudiantes o si el grupo es menor la mitad más uno, en total participaron 1.032 estudiantes. El segundo instrumento es un cuestionario que llena el investigador asistente ${ }^{1}$ que realiza en el aula de clase después de observar la práctica pedagógica.

Las variables seleccionadas fueron Rendimiento Académico (RA) y Estrategias pedagógicas (EP). Se generaron varios inter-grupos por facultades tabulándose estos resultados; posteriormente se

\footnotetext{
${ }^{1}$ Se agradece la participación de los estudiantes de seminario investigativo III de Ingeniería de Sistemas y de Estadística de Contaduría Pública apoyados por la Especialista Yesica M. Pérez
}

realizó un comparativo entre el rendimiento académico de los estudiantes y el promedio de las estrategias pedagógicas empleadas por estos entre las distintas facultades. Para realizar los comparativos de cada facultad de empleó el coeficiente de relación de Pearson (Pita \& Pértega, 2001) teniendo en cuenta como variable $X$ la opinión de los docentes y como variable $\mathrm{Y}$ la opinión de los estudiantes, empleando las siguientes fórmulas:

$$
\begin{aligned}
\rho_{X, Y} & =\frac{\sigma_{X Y}}{\sigma_{X} \sigma_{Y}}=\frac{E\left[\left(X-\mu_{X}\right)\left(Y-\mu_{Y}\right)\right]}{\sigma_{X} \sigma_{Y}} \\
r_{x y} & =\frac{\sum x_{i} y_{i}-n \bar{x} \bar{y}}{(n-1) S_{x} S_{y}} \\
= & \frac{n \sum x_{i} y_{i}-\sum x_{i} \sum y_{i}}{\sqrt{n \sum x_{i}^{2}-\left(\sum x_{i}\right)^{2}} \sqrt{n \sum y_{i}^{2}-\left(\sum y_{i}\right)^{2}}}
\end{aligned}
$$

Dónde:

$$
\begin{array}{ll}
\sigma_{X Y} & \text { Covarianza de }(X, Y) \\
\sigma_{X} & \text { Desviación típica de la variable } X \\
\sigma_{Y} & \text { Desviación típica de la variable } Y \\
r_{x y} & X \text { es estudiante, } Y \text { es docente }
\end{array}
$$

También se analiza el tipo de correlación que puede dar siendo relación directa si $r=1$ o también llamada positiva perfecta lo que indica una dependencia total entre las dos variables. Relación positiva si $0<r<1$; no existe relación lineal si $r=0$; relación negativa $-1<\mathrm{r}<0$ o la relación negativa perfecta si $r=-1$ cuando una aumenta la otra disminuye también llamada relación inversa.

\section{DESARROLLO DE LA INVESTIGACIÓN}

Como la idea de la investigación está enfocada a la evaluación de la adopción del modelo pedagógico de la universidad, que hasta el momento es el constructivista social con miras a evolucionar al dialógico-crítico (PEI-UFPS, 2007) en el cual "Se propone un enfoque pedagógico, dialógico y crítico, desde una perspectiva que asuma la práctica pedagógica como una cultura de paz; por lo tanto, se generarán políticas académicas que contribuyan a erradicar el simple transmisionismo de información y se privilegiarán aquellos modelos pedagógicos centrados en la construcción del conocimiento, a partir del diálogo permanente entre el maestro y su estudiante en torno a la ciencia, atendiendo la formación integral de nuestros profesionales". 
Se quiere medir en esta fase de la investigación cuales son las estrategias pedagógicas que los docentes de las cuatro facultades están adoptando y compararlas con el rendimiento académico que presentan los estudiantes. Esta revisión se realiza para el segundo semestre académico del 2015 con resultados parciales de los estudiantes (SIA, 2015) por tanto las variables seleccionadas fueron Rendimiento Académico (RA) y Estrategias pedagógicas (EP).

Se definieron las siguientes hipótesis:

Ho: Con la incorporación de estrategias pedagógicas (EP) por parte de los docentes de la universidad (DOC_UFPSO), se mejora el rendimiento académico (RA) de los estudiantes de la universidad Francisco de Paula Santander Ocaña EST_UFPSO.

H'o: Con la incorporación de estrategias pedagógicas (EP) por parte de los docentes de la universidad (DOC_UFPSO), NO se mejora el rendimiento académico (RA) de los estudiantes de la universidad Francisco de Paula Santander Ocaña EST UFPSO.

Dentro del plan de análisis se contempló:

1. Comparativo de las Estrategias pedagógicas empleadas en las diferentes facultades

2. Correlación de Pearson para docentes y estudiantes de la Facultad de Ingeniería.

3. Correlación de Pearson para docentes y estudiantes de la Facultad de Educación, Artes y Humanidades.

4. Correlación de Pearson para docentes y estudiantes de la Facultad de Ciencias Administrativas y Económicas.

5. Correlación de Pearson para docentes y estudiantes de la Facultad de Ciencias Agrarias.

6. Comparativo de Correlación de Pearson para las diferentes facultades.

7. Comparativo Rendimiento Académico y Estrategias pedagógicas teniendo en cuenta los tres actores docentes, estudiantes y el observador

\section{RESULTADOS}

\subsection{Comparativo de las Estrategias pedagógicas} empleadas en las diferentes facultades
Se toma la información dada por cada uno de los grupos docentes, estudiantes y el observador, se analiza por docente cada una de las estrategias seleccionadas siete en total, dando un valor porcentual para efectos de comparación.

De cada docente se revisa la información dada por sus estudiantes en cada una de las siete estrategias planteadas y se analiza según el observador a través de la escala de linkert, llevándolo al valor porcentual para realizar la comparación respectiva. E1 representa casos prácticos, E2 tecnología, E3 foros, E4 técnicas grupales, E5 diferentes tipos de evaluación, E6 diferentes formas de representaciones y E7 proyectos y análisis de problemáticas como las variables para la representación de las Estrategias empleadas.

Los resultados los podemos apreciar en la Tabla 1 comparativo de la Facultad de Ingeniería, en la Tabla 2 la Facultad de Ciencias Administrativas y Económicas, en la Tabla 3 los resultados de la facultad de Educación, Artes y Humanidades y por último se presentan los datos de la facultad de Ciencias Agrarias y del Medio Ambiente en la Tabla 4

Tabla 1 Comparativo porcentual apreciación Docentes y Estudiantes y observador Facultad de Ingeniería

\begin{tabular}{lrrrrrrrrr}
\hline F.INGENIERIA & E1 & E2 & E3 & E4 & E5 & E6 & E7 & TOTA. & PRO \\
\hline DOCENTES & 90 & 95 & 45 & 70 & 35 & 70 & 75 & 20 & 69 \\
ESTUDIANTES & 68 & 63 & 39 & 60 & 32 & 43 & 45 & 230 & 50 \\
OBSERVADOR & 78 & 79 & 66 & 78 & 58 & 78 & 80 & 20 & 74 \\
\hline
\end{tabular}

Como aspectos a resaltar la opinión de los estudiantes es la más baja está en promedio en un $50 \%$ respecto a la adopción de estas estrategias planteadas, en los docentes vemos unos resultados más altos casi un $70 \%$ semejante a los observadores que opinan que se están empleando en un $74 \%$.

Tabla 2: Comparativo porcentual apreciación Docentes y Estudiantes y observador Facultad de Ciencias Administrativas y Económicas

\begin{tabular}{lcccccccrr}
\hline F. CAE & E1 & E2 & E3 & E4 & E5 & E6 & E7 & TOTAL & PRO \\
\hline DOCENTES & 95 & 53 & 53 & 95 & 26 & 53 & 68 & 19 & 63 \\
ESTUDIANTES & 83 & 66 & 42 & 79 & 29 & 58 & 55 & 276 & 59 \\
OBSERVADOR & 78 & 66 & 72 & 73 & 55 & 76 & 69 & 19 & 70 \\
\hline
\end{tabular}

En la Facultad de Ciencias Administrativas y Económicas los estudiantes mejoran esa percepción casi en un $60 \%$ semejante a los docentes con un 
$63 \%$ y los observadores mantienen una buena opinión con un $70 \%$

Tabla 3: Comparativo porcentual apreciación Docentes y Estudiantes y observador Facultad de Educación, Artes y Humanidades

\begin{tabular}{lrrrrrrrrr}
\hline FEAH & E1 & E2 & E3 & E4 & E5 & E6 & E7 & TOTAL & PRO \\
\hline DOCENTES & 76 & 62 & 52 & 90 & 38 & 43 & 71 & 21 & 62 \\
ESTUDIANTES & 78 & 48 & 40 & 78 & 20 & 37 & 52 & 315 & 51 \\
OBSERVADOR & 71 & 79 & 87 & 76 & 83 & 84 & 82 & 21 & 80 \\
\hline
\end{tabular}

Baja nuevamente la opinión de los estudiantes en la Facultad de Educación, Artes y Humanidades casi por mitad en un 51\%, los docentes tienen una percepción mejor en un $62 \%$ y los observadores tiene una opinión alta en un $80 \%$ ven una buena adopción de estas estrategias.

Tabla 4: Comparativo porcentual apreciación Docentes y Estudiantes y observador Facultad de Ciencias Agrarias y del Medio Ambiente

\begin{tabular}{lrrrrrrrrr}
\hline FCAMA & E1 & E2 & E3 & E4 & E5 & E6 & E7 & TOTAL & PRO \\
\hline DOCENTES & 94 & 81 & 50 & 100 & 31 & 75 & 75 & 16 & 72 \\
ESTUDIANTES & 80 & 74 & 42 & 78 & 35 & 66 & 62 & 211 & 62 \\
OBSERVADOR & 62 & 57 & 60 & 67 & 50 & 53 & 62 & 16 & 59
\end{tabular}

Para los estudiantes de Ciencias agrarias es mejor su opinión está en un $62 \%$, sus docentes opinan que aplican estas estrategias en un $72 \%$ y los observadores acá tienen una opinión más baja en un $59 \%$.

\subsection{Correlación de Pearson para docentes y estudiantes de la Facultad de Ingeniería.}

En la Tabla 5 se aprecian las categorías analizadas, los puntajes de $r$ y el tipo de correlación. Cuando la correlación es mayor que cero o positiva, indica que ambas mediciones apuntan hacia la misma dirección, es decir tienen la misma tendencia. Para este caso dan positivas tanto para docentes como para estudiantes la opinión sobre la aplicación de las estrategias pedagógicas.

\section{Tabla 5: Correlación Docentes y Estudiantes Facultad de Ingeniería}

\begin{tabular}{lcl}
\hline CATEGORIAS & PUNTAE & $\begin{array}{l}\text { TIPO DE } \\
\text { CORRELACION }\end{array}$ \\
\hline CASOS PRACTICOS & 0,3372 & POSITIVA \\
TECNOLOGIA & 0,0761 & POSITIVA \\
FOROS & 0,4300 & POSITIVA \\
ACT. GRUPO & 0,0985 & POSITIVA \\
EVALUACION & 0,0597 & POSITIVA \\
REPRESENTACIONES & 0,4227 & POSITIVA \\
PROYECTOS & 0,3405 & POSITIVA \\
\hline
\end{tabular}

4.3 Correlación de Pearson para docentes y estudiantes de la Facultad de Educación, Artes y Humanidades.

En la Tabla 6 se aprecian los resultados aplicando el mismo método en la Facultad de Educación, Artes y Humanidades.

Tabla 6: Correlación Docentes y Estudiantes Facultad de Educación, Artes y Humanidades

\begin{tabular}{lrl}
\hline CATEGORIAS & PUNTAJE & $\begin{array}{l}\text { TIPO DE } \\
\text { CORREACION }\end{array}$ \\
\hline CASOS PRACTICOS & 0,3614 & POSITTVA \\
TECNOLOGIA & 0,1690 & POSITTVA \\
FOROS & 0,4254 & POSITTVA \\
ACT. GRUPO & $-0,0299$ & NEGATIVO \\
EVALUACION & 0,0140 & POSITTVA \\
REPRESENTACIONES & 0,3927 & POSITTVA \\
PROYECTOS & $-0,0443$ & NEGATIVO \\
\hline
\end{tabular}

En la figura 2 observamos dos resultados negativos o correlación negativa, mostrando que no hay dependencia entre las variables; en actividades grupales $y$ en proyectos o reflexión sobre problemáticas, el índice indica que no hay concordancia de opinión entre docentes y estudiantes en estas prácticas, lo podemos apreciar también en la figura 1.

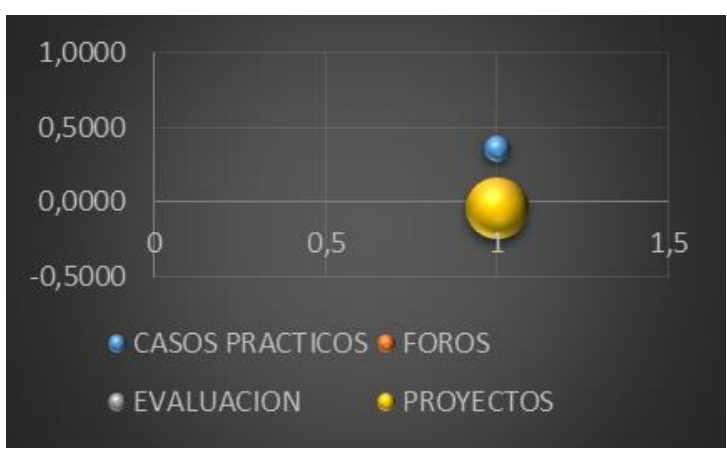

Fig. 1. Correlación de Pearson 
4.4 Correlación de Pearson para docentes y estudiantes de la Facultad de Ciencias Administrativas y Económicas.

Se observa la Tabla 7 después de hacer el análisis en la Facultad de Ciencias Administrativas y Económicas la opinión de sus docentes y estudiantes.

\begin{tabular}{|c|c|c|}
\hline \multicolumn{3}{|c|}{$\frac{\text { Facultad de Ciencias Administrativas y }}{\text { Económicas }}$} \\
\hline CATEGORIAS & PUNTAJE & $\begin{array}{l}\text { TIPO DE } \\
\text { CORRELACION }\end{array}$ \\
\hline CASOS PRACTICOS & 0,2540 & POSITTVA \\
\hline TECNOLOGLA & 0,5014 & POSITTVA \\
\hline FOROS & 0,3750 & POSITIVA \\
\hline ACT. GRUPO & $-0,2102$ & NEGATTVA \\
\hline EVALUACION & 0,6208 & POSITTVA \\
\hline REPRESENTACTONES & 0,2008 & POSITTVA \\
\hline PROYECTOS & 0,5258 & POSITIVA \\
\hline
\end{tabular}

Se presenta un resultado negativo en las actividades grupales o correlación negativa, mostrando que no hay dependencia entre las variables.

\subsection{Correlación de Pearson para docentes y estudiantes de la Facultad de Ciencias Agrarias.}

Se presenta en la Tabla 8 los resultados mostrando una correlación negativa en las actividades grupales, indicando que no hay dependencia entre las variables igual que en la facultad de Ciencias Administrativas y Económicas.

\begin{tabular}{lrl}
\multicolumn{3}{c}{ Tabla 8. Correlación Docentes y Estudiantes } \\
\multicolumn{3}{c}{ Facultad de Ciencias Agrarias y del Medio } \\
\hline \multicolumn{3}{c}{ Ambiente } \\
\hline CATEGORIAS & PUNTAJE & TIPO DE \\
& & CORRELACTON \\
\hline CASOS PRACTICOS & 0,6185 & POSITIVA \\
TECNOLOGIA & 0,6743 & POSITIVA \\
FOROS & 0,4181 & POSITIVA \\
ACT. GRUPO & $-0,0888$ & NEGATTVA \\
EVALUACION & 0,5690 & POSITIVA \\
REPRESENTACTONES & 0,6584 & POSITIVA \\
PROYECTOS & 0,4808 & POSITIVA \\
\hline
\end{tabular}

\subsection{Comparativo de Correlación de Pearson para las diferentes facultades.}

Para realizar el comparativo de correlación de Persona entre las facultades se consideró la opinión de los tres grupos docentes, estudiantes y el observador, dando como resultado una correlación positiva entre los mismos se pueden ver los resultados en la tabla 9.
Tabla 9. Comparativo correlación de Pearson

\begin{tabular}{lll}
\multicolumn{3}{c}{ entre facultades } \\
\hline CATEGORIAS & PUNTAJE & TIPO DE \\
& & CORRELACION \\
\hline NGENIERIA & 0,37209754 & POSITIVA \\
EDUCACION & 0,10336604 & POSITTVA \\
ADMINNTRATTVAS & 0,45104857 & POSITTVA \\
AGRARIAS & 0,64417545 & POSITTVA \\
\hline
\end{tabular}

También lo podemos observar de forma gráfica en el gráfico 2.

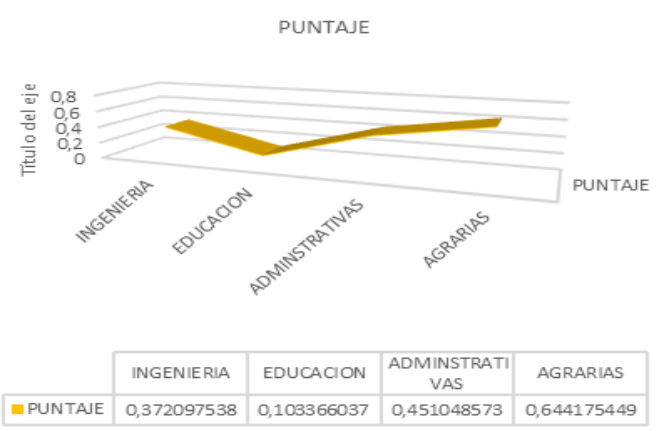

Fig. 2. Correlación de Pearson entre Facultades

4.7 Comparativo entre el rendimiento académico y estrategias pedagógicas

Por último en la tabla 10 se analizan los docentes el porcentaje establecido de estrategias empleadas, el promedio de las evaluaciones de los estudiantes en cuanto a su rendimiento académico y la edad del docente, para esto son tenidos en cuenta los docentes y estudiantes en su percepción sobre la adopción de las estrategias y para el rendimiento académico se tomó solo el primer parcial del sistema de información académico de la universidad.

\begin{tabular}{|c|c|c|c|}
\hline \multicolumn{4}{|c|}{ Estrategias pedagógicas } \\
\hline CATEGORIAS & $\begin{array}{c}\text { PORCENTAJE } \\
\text { ADOPCION } \\
\text { EP }\end{array}$ & $\begin{array}{l}\text { RENDIMIENTO } \\
\text { ACADEMICO }\end{array}$ & EDAD \\
\hline INGENIERIAS & 60,11 & 2,94 & 42,05 \\
\hline $\begin{array}{l}\text { EDUCACION } \\
\text { ARTESY }\end{array}$ & 61,90 & 3,34 & 42,12 \\
\hline $\begin{array}{l}\text { HUMANIDADES } \\
\text { CIENCIAS } \\
\text { ADMINISTRATIVAS } \\
\text { YECONOMICAS }\end{array}$ & 61,57 & 3,33 & 46,09 \\
\hline $\begin{array}{l}\text { CIENCIAS } \\
\text { AGRARIASY DEL } \\
\text { AMBIENTE }\end{array}$ & 68,31 & 2,89 & 44,69 \\
\hline
\end{tabular}

En el gráfico 3 se ve gráficamente la relación que se da entre porcentaje de adopción de Estrategias pedagógicas y el rendimiento académico parcial que llevan los estudiantes en el semestre. 
La facultad de Ingeniería y la de Educación, Artes y Humanidades muestra una relación positiva, en la facultad de ciencias Administrativas y Económicas se nota una pequeña diferencia pero los resultados son muy similares donde se ve una gran diferencia es en la facultad de Ciencias Agrarias y del Medio Ambiente donde los resultados muestran tendencias totalmente opuestas.

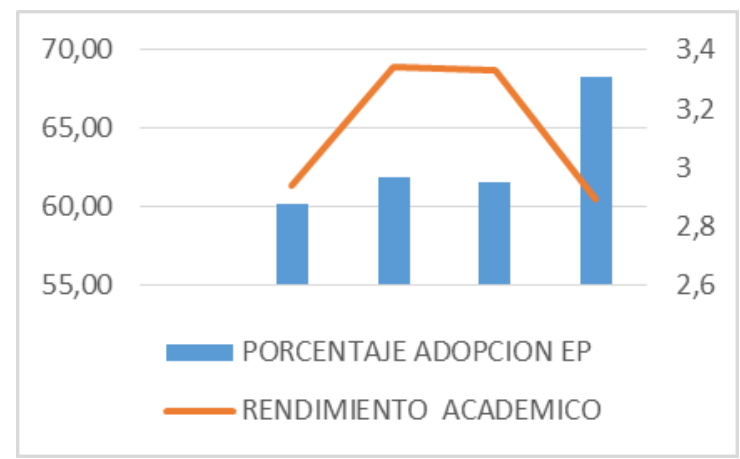

Fig. 3. Relación porcentaje de adopción de Estrategias pedagógicas y el rendimiento académico parcial

\section{CONCLUSIONES}

Al tomar los datos promedios de los tres grupos focales, en las cuatro facultades tendrían un valor promedio del $64 \%$, siendo esto un valor poco confiable ya que existen diferencias de opinión entre los tres grupos; pero si lo vemos por cada grupo, los estudiantes opinan que esta adopción esta por el orden del $56 \%$, los docentes en un $67 \%$ y los observadores en un $71 \%$. Al revisar por estrategias empleados vemos que las que consideran tienen más adopción son casos prácticos en un $80 \%$ y trabajos en grupo en un $79 \%$ y las menos adoptadas son los métodos de evaluación diversos en un $41 \%$ y los foros de discusión en un $54 \%$.

En el análisis de los resultados de los tres actores se ve una diferencia muy marcada en los observadores del proceso, lo que no representa un dato muy confiable para el análisis.

La facultad de Ingeniería mostró una correlación positiva en las variables de adopción de las estrategias pedagógicas tanto en docentes como estudiantes y al analizar en el comparativo de rendimiento académico versus estrategias se maneja la misma tendencia positiva.

En la facultad de Educación, Artes y Humanidades se dieron dos tendencias negativas en la opinión de los actores en actividades grupales y proyectos, pero con un valor bajo y al comparar su rendimiento académico se ve una relación entre las prácticas empleadas, caso muy parecido a la facultad de Ciencias Administrativas y Económicas que solo muestran una diferencia en las actividades grupales y también es muy parejo en su rendimiento académico.

En la facultad de Ciencias Agrarias hay una tendencia negativa en actividades grupales y se ve una diferencia sustancial en la percepción de los docentes y estudiantes respecto a la adopción de las estrategias que es la más alta de todas las facultades pero refleja el más bajo rendimiento académico, claro que estos resultados son parciales porque no se ha medido todo el semestre académico por tanto en estos momentos es difícil evaluar la hipótesis planteada.

Otras variables para tener en cuenta son la edad y el sexo, entre los docentes analizados existen 50 hombres y 26 mujeres, la edad promedio es de casi 44 años, lo que permite pensar en el perfil de los docentes de la universidad y como debe ser la forma de realizar en ellos procesos de cambio de modelos educativos, entendiéndose que la edad predominante según diversos autores se considera como la ideal para comprender la dinámica que les envuelve lo que permite el ajuste a las exigencias de la sociedad.

\section{RECOMENDACIONES}

La acción docente en la actualidad se complementa con un cúmulo de elementos que buscan en todo momento mejorar la calidad de los procesos de enseñanza y aprendizaje $\mathrm{y}$ dentro del enfoque constructivista se plantean diversas estrategias pedagógicas y didácticas cuya intención es aprovechar al máximo la creatividad de los estudiantes; buscando que la imaginación sea más productiva y que nutra los procesos garantizando una calidad en los mismos y un conocimiento sólido que se pueda extrapolar al diario acontecer de la sociedad. En tal sentido, el facilitador de los procesos pedagógicos se puede apoyar en herramientas didácticas que atraigan a los estudiantes y les genere curiosidad para que lleguen a ser exitosos en su desempeño laborar.

Es oportuno señalar que una de las estrategias pedagógicas a emplear pudiese ser el bucle recursivo planteado por Morir aplicando con las variantes necesarias de acuerdo al contenido, 
momento y contexto donde sea aplicado. Es significativo señalar que tal aplicación es precisa en el enfoque constructivista pues parte desde el desorden hasta consolidar la organización y de esa manera se nutre el constructivismo en la enseñanza. Otro de los aspectos que no se deben dejar a un lado es el uso y aplicación de los procesos cognitivos como sacar lo positivo, negativo, interesante (PNI) para la toma de decisiones, entre otros.

Aunado a las estrategias anteriores sería interesante aplicar la de-construcción como una manera de enseñanza puesto que se plantea que de acuerdo a la experiencia se pueden desmontar situaciones y generar nuevos conocimientos eso contribuye a que los futuros profesiones sean capaces de crear sus propios sistemas de solución de problemas cuando se les presente cualquier situación. Tal vez pudiese existir infinidad de estrategias, sin embargo cada una de ellas requiere de un análisis minucioso y detallado para su aplicabilidad puesto que debe encajar en el enfoque constructivista y es de reconfirma que su radio de acción va desde ir encajando, montando y construyendo conocimiento a partir de otros ya existente y es preciso señalar que cada peldaño debe ajustarse a los momentos de la didáctica (enganche, desarrollo y cierre) para evitar discrepancia en el momento de su aplicabilidad.

\section{REFERENCIAS}

Duran Chinchilla, C., Velasquez Pérez, T., \& Cardenas García, M. (septiembre de 2013). El modelo pedagógico de la UFPS y su relación con las prácticas educativas. Cartagena de Indias, Colombia: Asociación Colombiana de Ingenierías ACOFI.

Flórez, O. (1994). Hacia una Pedagogìa del Conocimiento. Colombia: McGraw Hill.

Jonassen, D. (1994). Thinking Technology: Toward a constructivist design model. Educational Technology.
OARC, (2015). Reporte de Matrícula, Oficina de Admisiones, Registro y Control Académico, Universidad Francisco de Paula Santander Ocaña. Reporte generado agosto 30 de 2.015.

PEI (2012). Proyecto Educativo Institucional. Universidad Francisco de Paula Santander Seccional Ocaña.

PEI-CUCUTA (2007). Proyecto Educativo Institucional. Universidad Francisco de Paula Santander.

SIA (2015). Sistema de Información Académico. División de Sistemas, Universidad Francisco de Paula Santander Ocaña. Reporte generado octubre 22 de 2.015.

Umanzor, P. (2011). El enfoque constructivista como estrategia para mejorar la calidad de la educación. PARADIGMA, revista de investigación educativa, 42-59.

Velásquez T. (2010). Evaluación De Los Aspectos

Curriculares Del Programa Ingeniería De Sistemas. Revista Colombiana de Tecnologías de Avanzada. ISSN: 1692-7257, Vol. 2, No. 16.

Araque G J., Díaz R J.L., Gualdrón G O.E. (2013). Optimización del THD en un convertidor multinivel monofásico usando algoritmos genéticos. Revista colombiana de tecnologías de Avanzada, Vol. 1, No. (21), pp. 60 - 66

Velásquez T. at et. (2014). Orientación vocacional aplicando sistemas basados en conocimiento. Revista Colombiana de Tecnologías de Avanzada, ISSN: 1692-7257, Vol. 1, No. 23.

Velásquez T. at et. (2015). A look at the ontoepistemic fundamentals of the carrer of System Engineering as a basis for rethinking the social impact in times of changing. Revista Colombiana de Tecnologías de Avanzada, ISSN: 1692-7257, Vol. 1, No. 25.

Velásquez, Pérez et al (2.015). Prácticas Pedagógicas en la Especialización de Auditoria de Sistemas. Congreso Internacional en Educación, Artes y Humanidades, 2015.Clymer, J. R. (1992). "Discrete Event Fuzzy Airport Control". IEEE Trans. on Systems, Man, and Cybernetics, V. 22, No. 2.

Wagner, R. (1.954) Probleme und BeispielebiologischerRegelung, Struffgart, Thieme. 